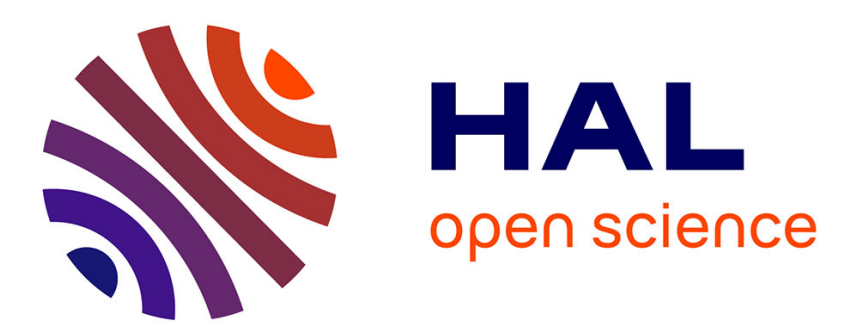

\title{
State and output feedback control for Takagi-Sugeno systems with saturated actuators
}

\author{
Souad Bezzaoucha, Benoît Marx, Didier Maquin, José Ragot
}

\section{To cite this version:}

Souad Bezzaoucha, Benoît Marx, Didier Maquin, José Ragot. State and output feedback control for Takagi-Sugeno systems with saturated actuators. International Journal of Adaptive Control and Signal Processing, 2016, 30 (6), pp.888-905. 10.1002/acs.2649 . hal-01248309

\section{HAL Id: hal-01248309 \\ https://hal.science/hal-01248309}

Submitted on 30 Nov 2021

HAL is a multi-disciplinary open access archive for the deposit and dissemination of scientific research documents, whether they are published or not. The documents may come from teaching and research institutions in France or abroad, or from public or private research centers.
L'archive ouverte pluridisciplinaire HAL, est destinée au dépôt et à la diffusion de documents scientifiques de niveau recherche, publiés ou non, émanant des établissements d'enseignement et de recherche français ou étrangers, des laboratoires publics ou privés. 


\title{
State and output feedback control for Takagi-Sugeno systems with saturated actuators
}

\author{
Souad Bezzaoucha ${ }^{1, *, \dagger}$, Benoît Marx ${ }^{2,3}$, Didier Maquin ${ }^{2,3}$ and José Ragot ${ }^{2,3}$ \\ 'INP Enseirb-Matmeca, Bordeaux Institute of Technology, IMS-lab, 351 cours de la libération, 33405 Talence, France \\ ${ }^{2}$ Lorraine University, CRAN, UMR 7039, 2 avenue de la forêt de Haye, Vandoeuvre-lès-Nancy Cedex, 54516, France
}

${ }^{3}$ CNRS, CRAN, UMR 7039, France

\begin{abstract}
SUMMARY
In the present paper, a Takagi-Sugeno (TS) model is used to simultaneously represent the behaviour of a nonlinear system and its saturated actuators. With the TS formalism and the Lyapunov approach, stabilization conditions are expressed as linear matrix inequalities for different controller designs. Static parallel distributed compensation (PDC) state feedback and static and dynamic PDC output feedback controllers for nonlinear saturated systems are proposed. The descriptor approach is used to obtain relaxed conditions to compute the controller gains. The nonlinear cart-pendulum system is used to illustrate the proposed control laws.
\end{abstract}

KEY WORDS: nonlinear system; TS model; actuator saturation; parallel distributed compensation design; descriptor approach; static and dynamic output feedback

\section{INTRODUCTION}

Actuator saturation or control input saturation is probably one of the most usual nonlinearity encountered in control engineering due to the physical impossibility of applying unbounded control signals and/or safety constraints. Actuator saturation may cause decreasing performances or even instability of the system under control, because the needed control input energy may not be provided to the system, for instance, when the control requires large gains resulting in control law magnitudes exceeding the range of the actuators. Motivated by these issues, some efforts have focused on developing saturated controllers for regulation problems [1-6].

There are two main design strategies to deal with actuator saturation. The first strategy is a twostep approach in which a nominal linear controller is first constructed by ignoring actuator saturation and using standard linear design tools; thereafter, a so-called anti-windup compensator is designed to handle the saturation constraints [7-9]. A typical anti-windup scheme consists in augmenting a nominal pre-designed linear controller with a compensator based on the discrepancy between unsaturated and saturated control signals provided to the plant [10]. A generalization of the antiwindup strategy based on a modified sector condition is used to obtain stability conditions from a quadratic Lyapunov function as proposed in [11]. However, for saturated linear parameter varying (LPV) systems, only few works are available on the subject, like [12-15] and [16] where the modified sector condition from [11] was applied to the LPV case under saturated inputs and states.

On the contrary, the second strategy consists in considering the saturation from the beginning of the controller design, and then the controller gains are adjusted accordingly to the saturation

\footnotetext{
*Correspondence to: Souad Bezzaoucha, INP Enseirb-Matmeca, Bordeaux Institute of Technology, IMS-lab, 351 cours de la libération, 33405 Talence, France.

†-mail: souad.bezzaoucha@u-bordeaux.fr
} 
levels. Among these strategies, one can cite the invariant sets framework, which has been significantly developed in control engineering over the last decades [17, 18]. This framework ensures that every state trajectory initialized inside a so-called invariant set remains inside this set. An interesting approach of the invariant set framework, applied in [19] and [20], consists in the so-called polytopic rewriting of the saturation constraint and is used to determine the largest invariant set by maximizing an estimate of the basin of attraction of the closed-loop system [21, 22]. Unlike in [23], where the occurrence of saturation is allowed, in most papers, the overall goal of the previous method could be roughly understood as preventing the controller from reaching the saturation constraints, implying a decrease of the control input energy possibly provided to the system. Contrarily, in the present contribution, the saturation is explicitly considered, and its occurrence is admitted. The Takagi-Sugeno (or polytopic; TS) modeling is here used to represent the saturation constraints and integrate them in the controller design in order to compute the controller gains according to the saturation levels and to ensure the closed-loop system stability.

The aim of this paper is to present a new approach for saturated control of nonlinear systems, where the sector nonlinearity transformation (SNT) is used to represent both the saturated actuators and the nonlinearities of the system itself under a TS form.

One should note that even if the expressions of the TS saturation are similar to the polytopic ones used in [19] and [20], the development, control strategy, and objectives are completely different. Indeed, in the proposed approach, the invariant sets are not considered and the objective concerns both the global stabilization of nonlinear systems represented with the TS models and the synthesis of a state feedback controller by parallel distributed compensation (PDC) with control gains explicitly depending on the saturation level.

After that, using only measured plant output signals, output feedback controllers are considered $[24,25]$. These controllers may be static or dynamic. Static output feedback control is the simplest approach because no further dynamics are introduced. However, a dynamic compensator introducing extra dynamics may be required to increase the number of degrees of freedom in the design and improve the closed-loop transient response. For this part, the descriptor approach is envisaged $[26,27]$. This approach is well known to avoid the coupling terms between the feedback gains and the Lyapunov matrices and thus facilitates the linear matrix inequalities (LMIs) resolution. As a consequence, the number of LMI decreases, and relaxed conditions are obtained [27, 28].

It is important to highlight that the proposed approach ensures the stability of nonlinear system that may be destabilized by the control saturation when the saturation is not taken into account in the control synthesis. Nevertheless, if the submodels are unstable (matrices $A_{i}$ of the TS model not stable), the proposed state and static output approaches are not suitable, but the dynamic output feedback controller (Section 5) can be used to stabilize the closed-loop system. The proposed dynamic control presents the advantage of stabilizing the closed-loop system with an additional degree of freedom in the design procedure which is the controller order (only the dimensions of the LMI variables are changed). This controller order may be set up according to a trade-off between reducing the controller order and the obtained closed-loop performance (assessed by the radius of the ball in which the state trajectory converges) for example.

The rest of this paper is organized as follows. The TS structure for modeling is first introduced in Section 2, also with some preliminary results, mathematical notations, and a brief description of the actuator saturation. Section 3 is devoted to the representation of the nonlinear saturation by a TS structure. In Section 4, the PDC-saturated state feedback control is detailed. The static and dynamic PDC output feedback controllers are studied in Section 5. To highlight the interest of the paper, the nonlinear model of a cart-pendulum is used, and stabilizing state and output feedback controllers is designed to counteract the effect of the actuator saturations.

\section{PRELIMINARIES}

The TS representation of a nonlinear system consists in a time-varying interpolation of a set of linear submodels. Each submodel contributes to the global behavior of the nonlinear system through a weighting function $h_{i}(\xi(t))[29]$. The TS structure is given by 


$$
\left\{\begin{array}{l}
\dot{x}(t)=\sum_{i=1}^{n} h_{i}(\xi(t))\left(A_{i} x(t)+B_{i} u(t)\right) \\
y(t)=\sum_{i=1}^{n} h_{i}(\xi(t))\left(C_{i} x(t)+D_{i} u(t)\right)
\end{array}\right.
$$

where $x(t) \in \mathbb{R}^{n_{x}}$ is the system state, $u(t) \in \mathbb{R}^{n_{u}}$ the control input, and $y(t) \in \mathbb{R}^{m}$ the system output. $\xi(t) \in \mathbb{R}^{q}$ is the premise variable assumed to be measurable or known signal (including at least the input $u$ and the output $y$ of the system, but also the state $x$ when it is accessible like in the state feedback case). The weighting functions $h_{i}(\xi(t))$, also noted $h_{i}(t)$, satisfy the so-called convex sum property

$$
\left\{\begin{array}{l}
\sum_{i=1}^{n} h_{i}(\xi(t))=1 \\
0 \leqslant h_{i}(\xi(t)) \leqslant 1, \quad i=1, \ldots, n
\end{array}\right.
$$

In the rest of the paper, the following lemmas are used.

Lemma 1 ([30])

For any matrices $X, Y$ and $G=G^{T}>0$ of appropriate dimensions, the following inequality holds

$$
X^{T} Y+Y^{T} X \leqslant X^{T} G X+Y^{T} G^{-1} Y
$$

Lemma 2 (congruence lemma, [30])

Consider two matrices $X$ and $Y$, if $X$ is positive (resp. negative) definite and if $Y$ is a full column rank matrix, then the matrix $Y^{T} X Y$ is positive (resp. negative) definite.

The following notations are used throughout the paper:

- A block diagonal matrix with the square matrices $A_{1}, \ldots, A_{n}$ on its diagonal is denoted as $\operatorname{diag}\left(A_{1}, \ldots, A_{n}\right)$.

- For any square matrix $M, \mathbb{S}(M)$ means $\mathbb{S}(M)=M+M^{T}$.

- The smallest and largest eigenvalues of the matrix $M$ are respectively denoted $\lambda_{\min }(M)$ and $\lambda_{\max }(M)$.

- The saturation function of the signal $u(t)$ is denoted by $\operatorname{sat}(u(t))$ and defined componentwise by (4) where $u_{\max }^{j}$ and $u_{\min }^{j}$ are the upper and lower saturation levels of the $j^{\text {th }}$ component of $u(t)$, denoted $u_{j}(t)$

$$
\operatorname{sat}\left(u_{j}(t)\right)= \begin{cases}u_{j}(t) & \text { if } u_{\min }^{j} \leqslant u(t) \leqslant u_{\max }^{j} \\ u_{\max }^{j} & \text { if } u_{j}(t)>u_{\max }^{j} \\ u_{\min }^{j} & \text { if } u_{j}(t)<u_{\min }^{j}\end{cases}
$$

\section{TS MODELING OF THE INPUT SATURATION}

The main idea of this work is to model the nonlinear actuator saturation using the TS representation and then propose PDC state and output feedbacks, as well as dynamic output feedback control law ensuring the stability of the closed-loop system. For that, it is proposed to re-write each saturated control input component (4) under the TS form (1) introduced in [31].

Let us consider a piecewise decomposition into three parts of each saturated actuator $\operatorname{sat}\left(u_{j}(t)\right)$ given by

$$
\operatorname{sat}\left(u_{j}(t)\right)=\sum_{i=1}^{3} \mu_{i}^{j}\left(u_{j}(t)\right)\left(\lambda_{i}^{j} u_{j}(t)+\gamma_{i}^{j}\right)
$$




$$
\text { with } \begin{aligned}
\lambda_{1}^{j} & =0 \lambda_{2}^{j}=1 \lambda_{3}^{j}=0 \\
\gamma_{1}^{j} & =u_{\min }^{j} \gamma_{2}^{j}=0 \gamma_{3}^{j}=u_{\max }^{j}
\end{aligned}
$$

and the activation functions

$$
\left\{\begin{array}{l}
\mu_{1}^{j}\left(u_{j}(t)\right)=\frac{1-\operatorname{sign}\left(u_{j}(t)-u_{\min }^{j}\right)}{2} \\
\mu_{2}^{j}\left(u_{j}(t)\right)=\frac{\operatorname{sign}\left(u_{j}(t)-u_{\min }^{j}\right)-\operatorname{sign}\left(u_{j}(t)-u_{\max }^{j}\right)}{2} \\
\mu_{3}^{j}\left(u_{j}(t)\right)=\frac{1+\operatorname{sign}\left(u_{j}(t)-u_{\max }^{j}\right)}{2}
\end{array}\right.
$$

where the sign function is defined as

$$
\operatorname{sign}(x):= \begin{cases}-1 & \text { if } x<0 \\ 0 & \text { if } x=0 \\ 1 & \text { if } x>0\end{cases}
$$

Note that in equation (5), $\operatorname{sat}\left(u_{j}(t)\right)$ depends on the activation functions $\mu_{i}^{j}\left(u_{j}(t)\right)$.

In order to lighten the notations, $\mu_{i}^{j}\left(u_{j}(t)\right)$ will be denoted as $\mu_{i}^{j}(t)$.

However, based on the convex sum property (2) of the activation functions (7), each control input vector component $u_{\text {sat }}^{j}$ can be written in order to have the same activation functions for all the input vector as

$$
\operatorname{sat}\left(u_{j}(t)\right)=\sum_{i=1}^{3} \mu_{i}^{j}(t)\left(\lambda_{i}^{j} u_{j}(t)+\gamma_{i}^{j}\right) \prod_{k=1, k \neq j}^{n_{u}} \sum_{j=1}^{3} \mu_{j}^{k}(t)
$$

Then, for $n_{u}$ inputs, $3^{n_{u}}$ submodels are obtained in the following compact form:

$$
\operatorname{sat}(u(t))=\sum_{i=1}^{3^{n_{u}}} \mu_{i}(t)\left(\Lambda_{i} u(t)+\Gamma_{i}\right)
$$

It is important to note that $\operatorname{sat}(u(t))(10)$ is directly expressed in terms of the control variable $u(t)$. The global weighting functions $\mu_{i}(t)$, the matrices $\Lambda_{i} \in \mathbb{R}^{n_{u} \times n_{u}}$, and vectors $\Gamma_{i} \in \mathbb{R}^{n_{u} \times 1}$ are defined as follows:

$$
\left\{\begin{aligned}
\mu_{i}(t) & =\prod_{j=1}^{n_{u}} \mu_{\sigma_{i}^{j}}^{j}(t) \\
\Lambda_{i} & =\operatorname{diag}\left(\lambda_{\sigma_{i}^{1}}^{1}, \ldots, \lambda_{\sigma_{i}^{n_{u}}}^{n_{n_{u}}}\right) \\
\Gamma_{i} & =\left[\gamma_{\sigma_{i}^{1}}^{1}, \ldots, \gamma_{\sigma_{i}^{n_{u}}}^{n_{u}}\right]^{T}
\end{aligned}\right.
$$

where the indexes $\sigma_{j}^{j}\left(i=1, \ldots, 3^{n_{u}}\right.$ and $j=1, \ldots, n_{u}$ ), equal to 1,2 or 3 , indicate which partition of the $j^{\text {th }}$ input $\left(\mu_{1}^{j}, \mu_{2}^{j}\right.$ or $\left.\mu_{3}^{j}\right)$ is involved in the $i^{\text {th }}$ submodel (see [31] for more details).

As a consequence, it is now possible to describe a nonlinear system with bounded inputs. For that purpose, let us now consider a TS system with actuator saturation:

$$
\left\{\begin{array}{l}
\dot{x}(t)=\sum_{j=1}^{n} h_{j}(\xi(t))\left(A_{j} x(t)+B_{j} \operatorname{sat}(u(t))\right) \\
y(t)=\sum_{j=1}^{n} h_{j}(\xi(t))\left(C_{j} x(t)+D_{j} \operatorname{sat}(u(t))\right)
\end{array}\right.
$$


According to the TS writing of the saturation (10), the system (12) can be written as

$$
\left\{\begin{array}{l}
\dot{x}(t)=\sum_{i=1}^{3^{n_{u}}} \sum_{j=1}^{n} \mu_{i}(t) h_{j}(\xi(t))\left(A_{j} x(t)+B_{j}\left(\Lambda_{i} u(t)+\Gamma_{i}\right)\right) \\
y(t)=\sum_{i=1}^{3^{n_{u}}} \sum_{j=1}^{n} \mu_{i}(t) h_{j}(\xi(t))\left(C_{j} x(t)+D_{j}\left(\Lambda_{i} u(t)+\Gamma_{i}\right)\right)
\end{array}\right.
$$

Remark 1

In the previous section, a TS representation of the saturation (10) has been proposed. It is important to highlight that this representation is directly expressed in terms of the control variable $u(t)$ and that the number of sub-models $\left(3^{n_{u}}\right)$ depends on the number of inputs $n_{u}$. This statement may introduce some conservatism. In fact, as the reader will notice in the following paper, the number of LMIs to solve to find the control gains depends on the number of sub-models used to describe the saturation constraint. As a first contribution, the saturation is expressed with a three-part piecewise decomposition, and decreasing the number of LMI conditions by finding more efficient TS representations of the control input saturation may be an interesting point for future works.

\section{SATURATED STATE FEEDBACK CONTROL LAW}

In this section, the system state is supposed to be known, and the objective is to design a state feedback control ensuring the stability of the closed-loop system, even in the presence of control input saturation. The control law is defined by a PDC state feedback:

$$
u(t)=-\sum_{j=1}^{n} h_{j}(\xi(t)) K_{j} x(t)
$$

The controller design is performed by the TS modeling of the saturation (10) and by solving an optimization problem under LMI constraints. In order to highlight the interest of considering the saturation when computing the controller, the controller design is envisaged without (Section 4.1) and with (Section 4.2) taking into account the control input bounds. A comparison of the obtained results is made in Section 4.3.

\subsection{Nominal control law (without saturation)}

In the nominal case, the controller gains $K_{j}$ are synthesized without taking into account the saturation limits. From (1) and (14), the closed-loop system is

$$
\dot{x}(t)=\sum_{i=1}^{n} \sum_{j=1}^{n} h_{i}(\xi(t)) h_{j}(\xi(t))\left(A_{i}-B_{i} K_{j}\right) x(t)
$$

In order to analyze the time evolution of the system state, using a quadratic Lyapunov function $V(x(t))=x^{T}(t) P^{-1} x(t)\left(P=P^{T}>0\right)$, it easily follows that the stability condition $\dot{V}(x(t))<0$ is ensured if there exists $P$ and $R_{j}$ such that the following LMI conditions hold [29]:

$$
\mathbb{S}\left(A_{i} P-B_{i} R_{j}\right)<0 \quad i=1, \ldots, n ; j=1, \ldots, n
$$

The gains of the controller (14) are then given by

$$
K_{j}=R_{j} P^{-1}, j=1, \ldots, n
$$




\subsection{Controller with saturation constraint}

In this section, the objective is to design a time-varying state feedback controller (14) to guarantee the stability of the bounded input system (12). Replacing (14) into (13) allows to express the dynamics of the system state as

$$
\dot{x}(t)=\sum_{i=1}^{n} \sum_{j=1}^{n} \sum_{k=1}^{3^{n u}} h_{i}(\xi(t)) h_{j}(\xi(t)) \mu_{k}(t)\left(\left(A_{i}-B_{i} \Lambda_{k} K_{j}\right) x(t)+B_{i} \Gamma_{k}\right)
$$

The controller synthesis consists in designing the gains $K_{j}$ ensuring the stability of the system (18) and the convergence of the state to an origin-centered ball as proposed in Theorem 1.

\section{Theorem 1}

There exists a time-varying state feedback controller (14) for a saturated input system (12) ensuring that the system state converges toward an origin-centered ball of radius bounded by $\beta$, if there exist matrices $P=P^{T}>0, R_{j}, \Sigma_{k}=\Sigma_{k}^{T}>0$ solutions of the following optimization problem (for $i=1, \ldots, n, j=1, \ldots, n$ and $k=1, \ldots, 3^{n_{u}}$ )

$$
\min _{P, R_{j}, \Sigma_{k}} \beta
$$

s.t.

$$
\begin{gathered}
\left(\begin{array}{cc}
Q_{i j k} & I \\
I & -\beta I
\end{array}\right)<0 \\
\Gamma_{k}^{T} B_{i}^{T} \Sigma_{k} B_{i} \Gamma_{k}<\beta
\end{gathered}
$$

with

$$
Q_{i j k}=\left(\begin{array}{cc}
\mathbb{S}\left(A_{i} P-B_{i} \Lambda_{k} R_{j}\right) & I \\
I & -\Sigma_{k}
\end{array}\right)
$$

The controller gains are given by

$$
K_{j}=R_{j} P^{-1}, \quad j=1, \ldots, n
$$

Proof

Let us define the following Lyapunov function

$$
V(x(t))=x^{T}(t) P^{-1} x(t)
$$

where $P=P^{T}>0$. According to equations (18) and (24), the time derivative of $V(x(t))$ is given by

$$
\dot{V}(x(t))=\sum_{i=1}^{n} \sum_{j=1}^{n} \sum_{k=1}^{3^{n u}} h_{i}(t) h_{j}(t) \mu_{k}(t)\left(\mathbb{S}\left(x^{T}(t) P^{-1} B_{i} \Gamma_{k}+x^{T}(t) P^{-1}\left(A_{i}-B_{i} \Lambda_{k} K_{j}\right) x(t)\right)\right)
$$

Using Lemma 1, with $\Sigma_{k}=\Sigma_{k}^{T}>0$, the time derivative of the Lyapunov function (25) is bounded as follows:

$$
\dot{V}(x(t)) \leqslant \sum_{i=1}^{n} \sum_{j=1}^{n} \sum_{k=1}^{3^{n_{u}}} h_{i}(t) h_{j}(t) \mu_{k}(t)\left(\Gamma_{k}^{T} B_{i}^{T} \Sigma_{k} B_{i} \Gamma_{k}+x^{T}(t) \mathscr{Q}_{i j k} x(t)\right)
$$


with

$$
\mathscr{Q}_{i j k}=\mathbb{S}\left(P^{-1}\left(A_{i}-B_{i} \Lambda_{k} K_{j}\right)\right)+P^{-1} \Sigma_{k}^{-1} P^{-1}
$$

Let us define

$$
\begin{gathered}
\varepsilon=\min _{i=1: n, j=1: n, k=1: 3^{n_{u}}} \lambda_{\min }\left(-\mathscr{Q}_{i j k}\right) \\
\delta=\max _{i=1: n, k=1: 3^{n_{u}}} \Gamma_{k}^{T} B_{i}^{T} \Sigma_{k} B_{i} \Gamma_{k}
\end{gathered}
$$

Because $\Sigma_{k}>0$ and from inequality (26), $\dot{V}(x(t))<-\varepsilon\|x(t)\|_{2}^{2}+\delta$. It follows that $\dot{V}(x(t))<0$ for

$$
\mathscr{Q}_{i j k}<0 \quad \text { and } \quad\|x(t)\|_{2}^{2}>\frac{\delta}{\varepsilon}
$$

which means that according to Lyapunov stability theory [32], $x(t)$ is uniformly bounded and converges to an origin-centered ball of radius $\sqrt{\frac{\delta}{\varepsilon}}$.

Let us now analyse the condition $\mathscr{Q}_{i j k}<0$.

Applying Lemma 2, this inequality becomes

$$
\mathbb{S}\left(\left(A_{i}-B_{i} \Lambda_{k} K_{j}\right) P\right)+\Sigma_{k}^{-1}<0
$$

Defining $R_{j}$ according to (23) and with a Schur complement, the inequalities (31) are equivalent to the $(1,1)$ block of $(20)$, that is, $Q_{i j k}<0$.

As the weighting functions $h_{i}(t), h_{j}(t), \mu_{k}(t)$ satisfy (2) and $\Sigma_{k}>0$, if $Q_{i j k}<0$ is satisfied for $i=1, \ldots, n, j=1, \ldots, n, k=1, \ldots, 3^{n_{u}}$, and $\|x\|_{2}^{2}>\frac{\delta}{\varepsilon}$, then $\dot{V}(x(t))<0$, implying that $x(t)$ converges to an origin-centered ball of radius $\sqrt{\frac{\delta}{\varepsilon}}$.

In order to improve the convergence to zero, the objective is now to minimize the radius $\sqrt{\frac{\delta}{\varepsilon}}$. Firstly, $\delta$ is bounded by $\beta$ from (29) and the LMIs (21). From (20), with a Schur complement, it obviously follows that

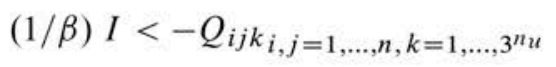

implying that all the eigenvalues of $\left(-Q_{i j k}\right)$ are larger that $1 / \beta$. As a consequence $1 / \beta<\varepsilon$ holds, and finally, the radius of the ball is bounded by $\beta$.

\subsection{Numerical example}

Let us consider a nonlinear model of the cart-pendulum system illustrated in Figure 1. The pendulum rotates in a vertical plan around an axis located on a cart. The cart can move along a horizontal rail, lying in the rotation plane. The characteristic variables of the system are $z(t)$ the cart position, $\dot{z}(t)$ the cart velocity, $\theta(t)$ the angle between the upward direction and the pendulum and $\dot{\theta}(t)$ the pendulum angular velocity. A control force $F(t)$ parallel to the rail is applied to the cart. The pendulum mass and cart mass are denoted $m=1 \mathrm{~kg}$ and $M=5 \mathrm{~kg}$, respectively. $l=0.1 \mathrm{~m}$ is the pendulum length and $I=510^{-3} \mathrm{kgm}^{2}$ the moment of inertia of the pendulum with respect to its rotation axis on the cart. The cart is subject to a viscous friction force $f \dot{z}(t)$, proportional to the cart velocity (with $f=100 \mathrm{Nm}^{-1} \mathrm{~s}$ ), to a static friction force $k_{s} z(t)$, proportional to the cart position (with $k_{s}=0.001 \mathrm{Nm}^{-1}$ ) and to a friction torque $k \dot{\theta}(t)$ proportional to the angular velocity (with $\left.k=0.045 \mathrm{Nrad}^{-1} \mathrm{~s}\right)$. 


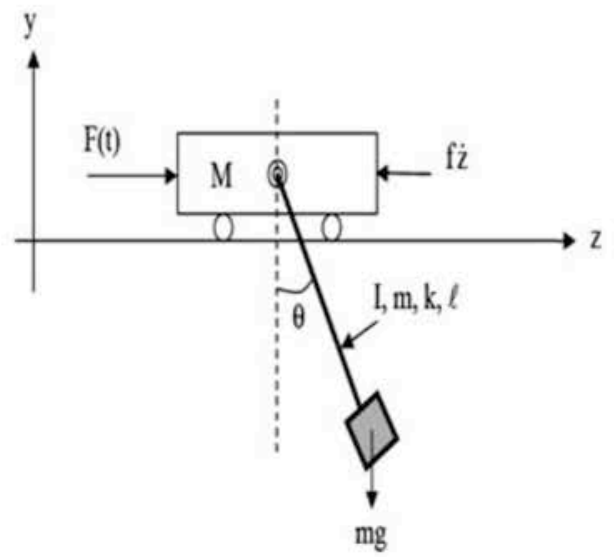

Figure 1. Cart-pendulum system.

Although the system may seem to be very academic, its popularity derives in part from the fact that it may be unstable with saturated control and non-null initial state. Additionally, the dynamics of the system are nonlinear.

For the sake of clarity, small angles are considered, and the nonlinear system may be simplified into

$$
\left\{\begin{array}{l}
(m+M) \ddot{z}(t)+k_{s} z(t)+f \dot{z}(t)-m l \ddot{\theta}(t)+m l \dot{\theta}^{2}(t) \theta(t)=F(t) \\
-m l \ddot{z}(t)+\left(m l^{2}+I\right) \ddot{\theta}(t)+k \dot{\theta}(t)+m g l \theta(t)=0
\end{array}\right.
$$

In order to ensure the stability of this nonlinear system and its state convergence to the origin-centred ball, first, the system equations (33) are written under a TS form by applying the SNT allowing to exactly represent the nonlinear system without any loss of information. Owing to space limitations, only the main steps to obtain the TS model are detailed; the interested reader can refer to [29] and in particular in section 2.2.1, examples 2 and 3, for more details.

First, the state variables and the control input are defined by

$$
x(t)=(z(t) \dot{z}(t) \theta(t) \dot{\theta}(t))^{T}, \quad u(t)=F(t)
$$

From (33) and (34), using factorization of $x$ and $u$, the following quasi-LPV form is deduced:

$$
\dot{x}(t)=A(x(t)) x(t)+B u(t)
$$

with

$$
A(x(t))=\left(\begin{array}{cccc}
0 & 1 & 0 & 0 \\
a_{1} & a_{2} & a_{3}(t) & a_{4} \\
0 & 0 & 0 & 1 \\
a_{5} & a_{6} & a_{7}(t) & a_{8}
\end{array}\right), B=\left(\begin{array}{c}
0 \\
b_{1} \\
0 \\
b_{2}
\end{array}\right)
$$

$$
\begin{aligned}
& b_{1}=\frac{1}{(m+M) \frac{(m)^{2}}{m l^{2}+I}} b_{2}=\frac{b_{1} m l}{m l^{2}+I} \quad a_{1}=-k_{s} b_{1} \quad a_{2}=-f b_{1} \quad a_{3}(t)=-b_{1} m l\left(g+x_{4}^{2}(t)\right) \\
& a_{4}=-\frac{b_{1} m l k}{m l^{2}+I} \quad a_{5}=\frac{a_{1} m l}{m l^{2}+I} \quad a_{6}=\frac{a_{2} m l}{m l^{2}+I} a_{7}(t)=\frac{a_{3}(t) m l}{m l^{2}+I}-g \quad a_{8}=\frac{a_{4} m l-k}{m l^{2}+I}
\end{aligned}
$$

Analyzing (37), the premise variable is chosen as $\xi(t)=x_{4}^{2}(t)$, which is bounded (owing to the angle and speed limitation). The SNT transformation is applied to obtain

$$
\xi(t)=h_{1}(t) \xi_{1}+h_{2}(t) \xi_{2}
$$




$$
\left\{\begin{array}{l}
\xi_{1}=\max \xi(t), \quad \xi_{2}=\min \xi(t) \\
h_{1}(t)=\frac{\xi(t)-\xi_{2}}{\xi_{1}-\xi_{2}}, \quad h_{2}(t)=1-h_{1}(t)
\end{array}\right.
$$

$h_{1}(t)$ and $h_{2}(t)$ represent the weighting functions of the TS model defined by

$$
\dot{x}(t)=\sum_{i=1}^{2} h_{i}(t)\left(A_{i} x(t)+B u(t)\right)
$$

From the definition of $\xi_{1}, \xi_{2}, h_{1}$ and $h_{2}$ in (39), it obviously follows that the convex sum properties $0 \leqslant h_{i}(t) \leqslant 1$ (for $\left.i=1,2\right)$ and $h_{1}(t)+h_{2}(t)=1$ are satisfied.

The matrices $A_{i}(i=1,2)$ are obtained by setting $a_{3}(t)$ respectively to $\left(-b_{1} m l\left(g+\xi_{1}\right)\right)$ and $\left(-b_{1} m l\left(g+\xi_{2}\right)\right)$ in $A(x(t))(36)$.

A state feedback (14) is considered both in the nominal (without saturation) and the saturated cases with respective gains $K_{i, N}$ and $K_{i, T S}$. From relations (16) and (17), the calculated control gains for the nominal case are equal to

$$
\begin{aligned}
& K_{1, N}=\left(\begin{array}{lll}
11.53 & -79.84 & 14.346 .48
\end{array}\right) \\
& K_{2, N}=\left(\begin{array}{lll}
9.95 & -82.1711 .785 .51
\end{array}\right)
\end{aligned}
$$

The gains $K_{i, T S}$, computed by solving the LMIs of the theorem 1 with $u_{\min }=0$ and $u_{\max }=3$, are given by

$$
\begin{aligned}
& K_{1, T S}=\left(\begin{array}{llll}
0.43 & 2.14 & 1.17 & 0.05
\end{array}\right) \\
& K_{2, T S}=\left(\begin{array}{llll}
0.37 & -9.19 & -0.10 & 0.53
\end{array}\right)
\end{aligned}
$$

A fourth control strategy is performed in order to compare a conventional anti-windup controller with the proposed one. For the conventional anti-windup, the main idea is to synthesize a nominal feedback control and to add a compensator to handle the saturated input. The anti-windup compensator is taken as a large gain matrix $\Xi=\alpha I$ with $\alpha=5$.

For the initial condition $x_{0}=(00 \pi / 120)^{T}$, Figure 2 shows the time evolution of the states where

- $x_{N}$ is the state trajectory obtained by applying the unsaturated nominal control. It is ruled by $\dot{x}_{N}(t)=\sum_{i=1}^{2} h_{i}(t)\left(A_{i}-B K_{i, N}\right) x_{N}(t)$.
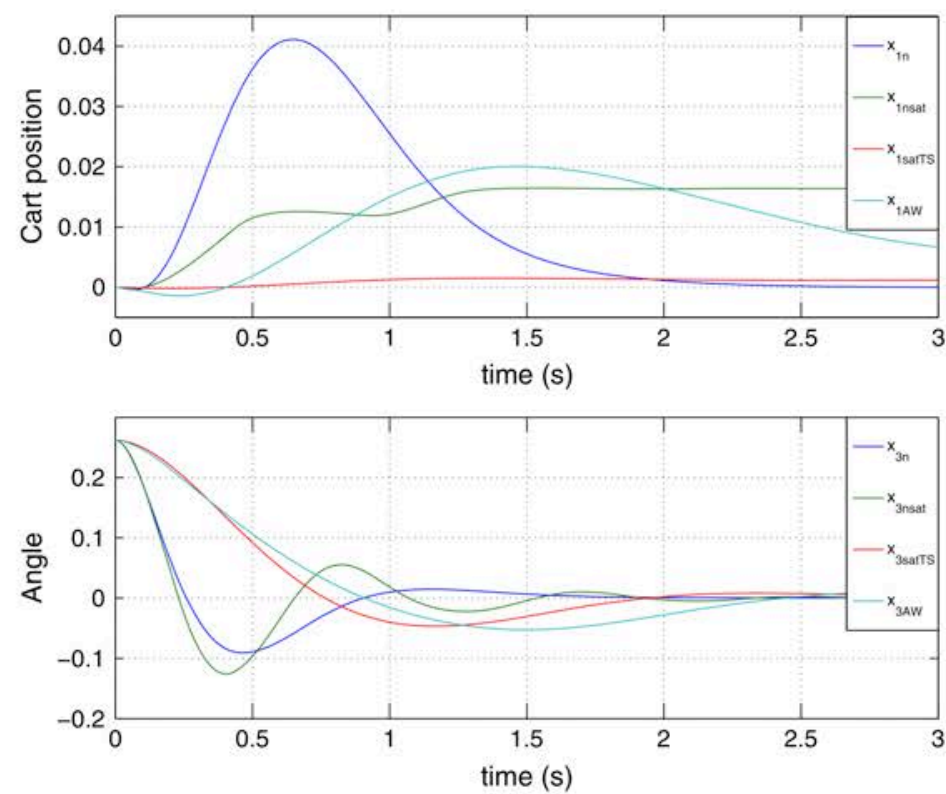

Figure 2. System states with state feedback control. 


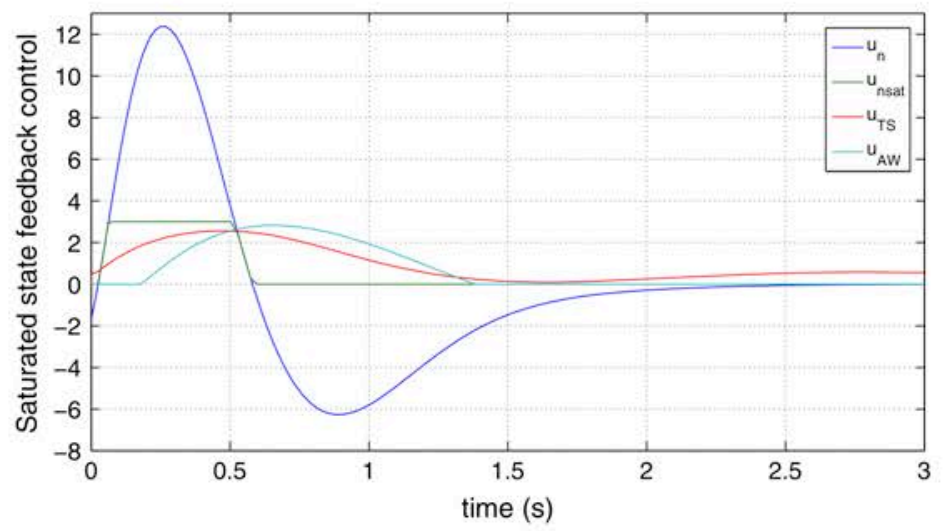

Figure 3. Saturated state feedback control.

- $x_{N, s a t}$ is the state trajectory obtained by applying the saturated nominal control. It is ruled by $\dot{x}_{N, s a t}(t)=\sum_{i=1}^{2} h_{i}(t) A_{i} x_{N, s a t}(t)+B \operatorname{sat}\left(-\sum_{i=1}^{2} h_{i}(t) K_{i, N} x_{N, s a t}(t)\right)$.

- $x_{s a t, T S}$ is the state trajectory obtained by applying the controller designed in the theorem 1. It is ruled by $\dot{x}_{s a t, T S}(t)=\sum_{i=1}^{2} h_{i}(t) A_{i} x_{s a t, T S}(t)+B s a t\left(-\sum_{i=1}^{2} h_{i}(t) K_{i, T S} x_{s a t, T S}(t)\right)$.

- $x_{A W}$ is the state obtained when applying the nominal control with an anti-windup module. The control input is adjusted using the difference $u_{\text {sat }}(t)-u(t)$, but the controller gain is computed without taking into account the saturation on the input control.

It can be seen on Figure 2 that the saturation of the nominal control prevent the first state variable of $x_{N, s a t}$ from converging close to the origin, whereas the proposed approach allows $x_{s a t, T S}$ to do so. It can also be seen that the transient state response obtained with the proposed controller is better damped than the one with the anti-windup (AW) controller.

The four nominal and saturated control signals are displayed on Figure 3

\section{OUTPUT FEEDBACK CONTROLLER: A DESCRIPTOR APPROACH}

The state feedback controller, proposed in the previous section, allows to efficiently compensate the input saturation, but it needs the whole state vector to be accessible. In this section, this limitation is overcome by envisaging output feedback control: if the state vector is not entirely available, static or dynamic output feedback controllers can be designed using only measured signal.

Static output feedback control is the simplest approach because no further dynamics are needed. However, a dynamic compensator introducing extra dynamics may be required to increase the number of freedom degrees in the design and improve the closed-loop transient response. It may be noted that dynamic output controller encompasses the class of observer-based controllers.

In the following section, both static and dynamic output feedback controllers are proposed in a unified framework, thanks to the descriptor approach. Sufficient LMI constraints are derived from the Lyapunov stability theory. Compared with [33], in the present paper, additional degrees of freedom are introduced in the Lyapunov function to obtain relaxed controller design conditions.

\subsection{Static feedback controller}

A PDC static output feedback control is envisaged:

$$
u(t)=\sum_{j=1}^{n} h_{j}(\xi(t)) K_{j}^{s} y(t)
$$

The proposed approach relies on a descriptor formulation, which is well known to avoid the coupling terms between the feedback gains and the Lyapunov matrices. As a consequence, the number of LMI decreases, and relaxed conditions are obtained [27]. 
The control law (43) and the system (12) are written as a descriptor system

$$
E^{s} \dot{x}_{a}(t)=\sum_{i=1}^{3^{n u}} \sum_{j=1}^{n} \mu_{i}(t) h_{j}(t)\left(\mathscr{A}_{i j}^{s} x_{a}(t)+\mathscr{B}_{i j}^{s}\right)
$$

where the augmented state vector and system matrices are defined by $x_{a}(t)=$ $\left(x^{T}(t) u^{T}(t) y^{T}(t)\right)$ and

$$
E^{s}=\operatorname{diag}\left(I_{n_{x}}, 0_{n_{u}+m}\right), \quad \mathscr{A}_{i j}^{s}=\left(\begin{array}{ccc}
A_{j} & B_{j} \Lambda_{i} & 0 \\
0 & -I_{n_{u}} & K_{j}^{s} \\
C_{j} & D_{j} \Lambda_{i} & -I_{m}
\end{array}\right), \quad \mathscr{B}_{i j}^{s}=\left(\begin{array}{c}
B_{j} \Gamma_{i} \\
0 \\
D_{j} \Gamma_{i}
\end{array}\right)
$$

The objective is to compute the controller gains $K_{j}^{s}$ of (43), according to the saturation limits, in order to guarantee the stability of the closed-loop system (44) .

\section{Theorem 2}

There exists a static feedback controller (43) for a system with bounded inputs (12) such that the system state converges toward an origin-centered ball of radius bounded by $\beta_{s}$, if there exist matrices $P_{1}^{s} \in \mathbb{R}^{n_{x} \times n_{x}}, P_{1}^{s}=\left(P_{1}^{s}\right)^{T}>0, P_{2}^{s} \in \mathbb{R}^{n_{u} \times n_{u}}, P_{2}^{s}>0, P_{31}^{s} \in \mathbb{R}^{m \times n_{x}}, P_{32}^{s} \in \mathbb{R}^{m \times n_{u}}, P_{33}^{s} \in$ $\mathbb{R}^{m \times m}, R_{j}^{s} \in \mathbb{R}^{n_{u} \times m}, \Sigma_{i j}^{1 s} \in \mathbb{R}^{n_{x} \times n_{x}}, \Sigma_{i j}^{1 s}=\left(\Sigma_{i j}^{1 s}\right)^{T}>0, \Sigma_{i j}^{3 s} \in \mathbb{R}^{m \times m}, \Sigma_{i j}^{3 s}=\left(\Sigma_{i j}^{3 s}\right)^{T}>0$, solutions of the following optimization problem (for $i=1, \ldots, 3^{n_{u}}$ and $j=1, \ldots, n$ )

$$
P_{1}^{s}, P_{2}^{s}, P_{31}^{s}, P_{32}^{s}, P_{33}^{s}, R_{j}^{s}, \Sigma_{i j}^{1 s}, \Sigma_{i j}^{3 s} \beta_{s}
$$

under the LMI constraints (46) and (47)

$$
\left.\begin{array}{ccc|cc|ccc}
Q_{i j}^{s 1} & Q_{i j}^{s 12} & C_{j}^{T} P_{33}^{s}-P_{31}^{s T} & P_{1}^{s} & P_{31}^{s T} & I_{n_{x}} & 0 & 0 \\
* & Q_{i j}^{s 2} & R_{j}^{s}+\Lambda_{i} D_{j}^{T} P_{33}^{s}-P_{32}^{s T} & 0 & P_{32}^{s T} & 0 & I_{n_{u}} & 0 \\
* & * & -P_{33}^{s}-\left(P_{33}^{s}\right)^{T} & 0 & P_{33}^{s T} & 0 & 0 & I_{m} \\
\hline * & * & * & -\Sigma_{i j}^{1 s} & 0 & 0 & 0 & 0 \\
* & * & * & * & -\Sigma_{i j}^{3 s} & 0 & 0 & 0 \\
\hline * & * & * & * & * & -\beta_{s} I_{n_{x}} & 0 & 0 \\
* & * & * & * & * & * & -\beta_{s} I_{n_{u}} & 0 \\
* & * & * & * & * & * & * & -\beta_{s} I_{m}
\end{array}\right)<0
$$

with

$$
\begin{aligned}
Q_{j}^{s 1} & =\mathbb{S}\left(P_{1}^{s} A_{j}+C_{j}^{T} P_{31}^{s}\right), Q_{i j}^{s 12} P_{1}^{s} B_{j} \Lambda_{i}+C_{j}^{T} P_{32}^{s}+P_{31}^{s T} D_{j} \Lambda_{i}^{T} \\
\text { and } \quad Q_{i j}^{s 2} & =\mathbb{S}\left(-P_{2}^{s}+P_{32}^{s T} D_{j} \Lambda_{i}\right)
\end{aligned}
$$

The controller gains are given by $K_{j}^{s}=\left(\left(P_{2}^{s}\right)^{T}\right)^{-1} R_{j}^{s}, j=1, \ldots, n$.

Proof

Let us define the Lyapunov function

$$
V\left(x_{a}(t)\right)=x_{a}^{T}(t)\left(E^{s}\right)^{T} P^{s} x_{a}(t)
$$


with $P^{s}$ defined by

$$
P^{s}=\left(\begin{array}{ccc}
P_{1}^{s} & 0 & 0 \\
0 & P_{2}^{s} & 0 \\
P_{31}^{s} & P_{32}^{s} & P_{33}^{s}
\end{array}\right)
$$

where $P_{1}^{s}=\left(P_{1}^{s}\right)^{T}>0$ and $P_{2}^{s}>0$. It follows from (50) and the structure of $E$ that $\left(E^{s}\right)^{T} P^{s}=$ $\left(P^{s}\right)^{T} E^{s} \geqslant 0$, and that the Lyapunov function is in fact quadratic in the state vector: $V\left(x_{a}(t)\right)=$ $x^{T}(t) P_{1}^{s} x(t)$.

Using the state equation (44), the time derivative of the Lyapunov function (49) is given by

$$
\dot{V}\left(x_{a}(t)\right)=\sum_{i=1}^{3^{n_{u}}} \sum_{j=1}^{n} \mu_{i}(t) h_{j}(t) \mathbb{S}\left(x_{a}^{T}(t)\left(P^{s}\right)^{T} \mathscr{B}_{i j}^{s}+x_{a}^{T}(t)\left(\mathscr{A}_{i j}^{s}\right)^{T} P^{s} x_{a}(t)\right)
$$

Using Lemma $1, \dot{V}\left(x_{a}(t)\right)$ is bounded as follows

$$
\dot{V}\left(x_{a}(t)\right) \leqslant \sum_{i=1}^{3^{n_{u}}} \sum_{j=1}^{n} \mu_{i}(t) h_{j}(t)\left(\Gamma_{i}^{T} B_{j}^{T} \Sigma_{i j}^{1 s} B_{j} \Gamma_{i}+\Gamma_{i}^{T} D_{j}^{T} \Sigma_{i j}^{3 s} D_{j} \Gamma_{i}+x_{a}^{T}(t) \mathscr{Q}_{i j}^{s} x_{a}(t)\right)
$$

with

$$
\begin{aligned}
& \mathscr{Q}_{i j}^{s}=\left(\mathscr{A}_{i j}^{s}\right)^{T} P^{s}+\left(P^{s}\right)^{T} \mathscr{A}_{i j}^{s}+\operatorname{diag}\left(P_{1}^{s}\left(\Sigma_{i j}^{1 s}\right)^{-1} P_{1}^{s}, 0,0\right) \\
& +\left(\begin{array}{lll}
P_{31}^{s} & P_{32}^{s} & P_{33}^{s}
\end{array}\right)^{T}\left(\Sigma_{i j}^{3 s}\right)^{-1}\left(\begin{array}{lll}
P_{31}^{s} & P_{32}^{s} & P_{33}^{s}
\end{array}\right)
\end{aligned}
$$

Let us define

$$
\begin{gathered}
\varepsilon^{s}=\min _{i=1: 3^{n^{u}}, j=1: n} \lambda_{\min }\left(-\mathscr{Q}_{i j}^{s}\right) \\
\delta^{s}=\max _{i=1: 3^{n_{u}}, j=1: n}\left(\Gamma_{i}^{T}\left(B_{j}^{T} \Sigma_{i j}^{1 s} B_{j}+D_{j}^{T} \Sigma_{i j}^{3 s} D_{j}\right) \Gamma_{i}\right)
\end{gathered}
$$

Because $\Sigma_{i j}^{1 s}$ and $\Sigma_{i j}^{3 s}$ are positive definite, from (52) with the convex sum property (2), $\dot{V}\left(x_{a}(t)\right)<$ $-\varepsilon^{s}\left\|x_{a}\right\|_{2}^{2}+\delta^{s}$. It follows that $\dot{V}\left(x_{a}(t)\right)<0$ for

$$
\mathscr{Q}_{i j}^{s}<0 \text { and }\left\|x_{a}\right\|_{2}^{2}>\frac{\delta^{s}}{\varepsilon^{s}}
$$

which means that $x_{a}(t)$ is uniformly bounded and converges to the origin-centered ball of radius $\sqrt{\delta^{s} / \varepsilon^{s}}$ according to Lyapunov stability theory [32]. Equation (46) is proved with some Schur's complements applied to $\mathscr{Q}_{i j}^{s}<0$ and the variable change $R_{j}^{s}=\left(P_{2}^{s}\right)^{T} K_{j}^{s}$. The objective is now to minimize the radius $\sqrt{\delta^{s} / \varepsilon^{s}}$. Firstly, using definition (55), $\delta^{s}$ is bounded by $\beta_{s}$ when considering LMIs (47). Secondly, it can be shown that $1 / \varepsilon^{s}<\beta_{s}$. From (54), it follows that

$$
-\mathscr{Q}_{i j}^{s}>\left(1 / \beta_{s}\right) I, i=1, \ldots, 3^{n_{u}}, j=1, \ldots, n
$$

meaning that all eigenvalues of $\left(-\mathscr{Q}_{i j}^{s}\right)$, including $\epsilon^{s}$, are bigger then $1 / \beta_{s}$. Thus, $1 / \epsilon^{s}<\beta_{s}$ and the radius $\sqrt{\delta^{s} / \varepsilon^{s}}$ is bounded by $\beta_{s}$.

\subsection{Dynamic output feedback controller}

The objective is now to design a stabilizing dynamic output feedback control even in the presence of control input saturation. As previously discussed, the solution is obtained by representing the 
saturation as a TS system and by solving an optimization problem under LMI constraints. Let us consider the following $n_{c}^{\text {th }}$ order dynamic output feedback controller defined by

$$
\left\{\begin{array}{l}
\dot{x}_{c}(t)=\sum_{j=1}^{n} h_{j}(t)\left(A_{j}^{c} x_{c}(t)+B_{j}^{c} y(t)\right) \\
u(t)=\sum_{j=1}^{n} h_{j}(t)\left(C_{j}^{c} x_{c}(t)+D_{j}^{c} y(t)\right)
\end{array}\right.
$$

designed to guarantee the stability of the saturated system (13). The matrices $A_{j}^{c} \in \mathbb{R}^{n_{c} \times n_{c}}, B_{j}^{c} \in$ $\mathbb{R}^{n_{c} \times m}, C_{j}^{c} \in \mathbb{R}^{n_{u} \times n_{c}}$ and $D_{j}^{c} \in \mathbb{R}^{n_{u} \times m}$ are the controller gains, determined to ensure the stability of the closed-loop system (13) with (58). The controller order $n_{c}$ can be adapted according to the control objectives and system dynamics. The closed-loop system defined from (13) and (58) is written under the following descriptor form:

$$
E^{d} \dot{x}_{a}(t)=\sum_{i=1}^{3^{n_{u}}} \sum_{j=1}^{n} \mu_{i}(t) h_{j}(t)\left(\mathscr{A}_{i j}^{d} x_{a}(t)+\mathscr{B}_{i j}^{d}\right)
$$

with $x_{a}(t)=\left(x^{T}(t) x_{c}^{T}(t) u^{T}(t) y^{T}(t)\right)$ and

$$
\mathscr{A}_{i j}^{d}=\left(\begin{array}{cccc}
A_{j} & 0 & B_{j} \Lambda_{i} & 0 \\
0 & A_{j}^{c} & 0 & B_{j}^{c} \\
0 & C_{j}^{c} & -I_{n_{u}} & D_{j}^{c} \\
C_{j} & 0 & D_{j} \Lambda_{i} & -I_{m}
\end{array}\right), \mathscr{B}_{i j}^{d}=\left(\begin{array}{c}
B_{j} \Gamma_{i} \\
0 \\
0 \\
D_{j} \Gamma_{i}
\end{array}\right), E^{d}=\left(\begin{array}{cc}
I_{n_{x}+n_{c}} & 0 \\
0 & 0_{n_{u}+m}
\end{array}\right)
$$

Theorem 3

There exists a dynamic feedback controller (58) for the saturated input system (12) such that the system state converges toward an origin-centered ball of radius bounded by $\beta_{d}$, if there exist matrices $P_{11}^{d} \in \mathbb{R}^{n_{x} \times n_{x}}, P_{11}^{d}=\left(P_{11}^{d}\right)^{T}>0, P_{22}^{d} \in \mathbb{R}^{n_{c} \times n_{c}}, P_{22}^{d}=\left(P_{22}^{d}\right)^{T}>0, P_{33}^{d} \in \mathbb{R}^{n_{u} \times n_{u}}>0$, $P_{41}^{d} \in \mathbb{R}^{m \times n_{x}}, P_{42}^{d} \in \mathbb{R}^{m \times n_{c}}, P_{43}^{d} \in \mathbb{R}^{m \times n_{u}}, P_{44}^{d} \in \mathbb{R}^{m \times m}, \bar{A}_{j}^{c} \in \mathbb{R}^{n_{c} \times n_{c}}, \bar{B}_{j}^{c} \in \mathbb{R}^{n_{c} \times m}$, $\bar{C}_{j}^{c} \in \mathbb{R}^{n_{u} \times n_{c}}, \bar{D}_{j}^{c} \in \mathbb{R}^{n_{u} \times m}, \Sigma_{i j}^{1 d}=\left(\Sigma_{i j}^{1 d}\right)^{T}>0$, and $\Sigma_{i j}^{2 d}=\left(\Sigma_{i j}^{2 d}\right)^{T}>0$, solutions of the following optimization problem (for $i=1, \ldots, 3^{n_{u}}$ and $j=1, \ldots, n$ )

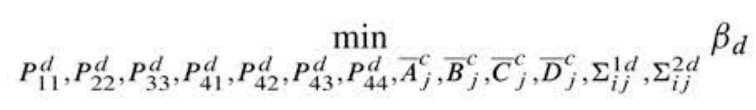

under the LMI constraints (62) and (63).

$$
\left.\begin{array}{cccc|cc|cccc}
Q_{i j}^{1} & C_{j}^{T} P_{42}^{d} & Q_{i j}^{13} & C_{j}^{T} P_{44}^{d}-\left(P_{41}^{d}\right)^{T} & P_{11}^{d} & \left(P_{41}^{d}\right)^{T} & I & 0 & 0 & 0 \\
* & \bar{A}_{j}^{c}+\left(\bar{A}_{j}^{c}\right)^{T} & \left(\bar{C}_{j}^{c}\right)^{T}+\left(P_{42}^{d}\right)^{T} D_{j} \Lambda_{i} & \overline{B_{j}^{c}-\left(P_{42}^{d}\right)^{T}} & 0 & \left(P_{42}^{d}\right. & 0 & I & 0 & 0 \\
* & * & Q_{i j}^{3} & \bar{D}_{j}^{c}-\left(P_{43}^{d}\right)^{T}+\Lambda_{i} D_{j}^{T} P_{44}^{d} & 0 & \left(P_{43}^{d}\right. & 0 & 0 & I & 0 \\
* & * & * & -P_{44}^{d}-\left(P_{44}^{d}\right)^{T} & 0 & \left(P_{44}^{d}\right)^{T} & 0 & 0 & 0 & I \\
\hline * & * & * & * & -\Sigma_{i j}^{I d} & 0 & 0 & 0 & 0 & 0 \\
* & * & * & * & * & -\Sigma_{i j}^{2 d} & 0 & 0 & 0 & 0 \\
\hline * & * & * & * & * & * & -\beta_{d} I & 0 & 0 & 0 \\
* & * & * & * & * & * & * & -\beta_{d} I & 0 & 0 \\
* & * & * & * & * & * & * & * & -\beta_{d} I & 0 \\
* & * & * & * & * & * & * & * & * & -\beta_{d} I
\end{array}\right)_{(62)}
$$


with

$$
\begin{aligned}
& Q_{i j}^{1}=A_{j}^{T} P_{11}^{d}+P_{11}^{d} A_{j}+C_{j}^{T} P_{41}^{d}+\left(P_{41}^{d}\right)^{T} C_{j} \\
& Q_{i j}^{13}=P_{11}^{d} B_{j} \Lambda_{i}+C_{j}^{T} P_{43}^{d}+\left(P_{41}^{d}\right)^{T} D_{j} \Lambda_{i} \\
& Q_{i j}^{3}=-P_{33}^{d}-\left(P_{33}^{d}\right)^{T}+\left(P_{43}^{d}\right)^{T} D_{j} \Lambda_{i}+\Lambda_{i} D_{j}^{T} P_{43}^{d}
\end{aligned}
$$

The gains of the controller (58) are given by

$$
\left\{\begin{array}{l}
A_{j}^{c}=\left(P_{22}^{d}\right)^{-1} \bar{A}_{j}^{c} \quad B_{j}^{c}=\left(P_{22}^{d}\right)^{-1} \bar{B}_{j}^{c} \\
C_{j}^{c}=\left(\left(P_{33}^{d}\right)^{-1}\right)^{T} \bar{C}_{j}^{c} D_{j}^{c}=\left(\left(P_{33}^{d}\right)^{-1}\right)^{T} \bar{D}_{j}^{c}
\end{array}\right.
$$

Proof

Let us define the Lyapunov function $V\left(x_{a}(t)\right)=x_{a}^{T}(t)\left(E^{d}\right)^{T} P^{d} x_{a}(t)$ with $E^{d}$ and $P^{d}$, respectively, defined in (60) and (66) that satisfy

$$
\left(E^{d}\right)^{T} P^{d}=\left(P^{d}\right)^{T} E^{d} \geqslant 0
$$

The matrix $P^{d}$ is partitioned according to $\mathscr{A}_{i j}^{d}$ defined in (60), and for satisfying (65), it is chosen as

$$
P^{d}=\left(\begin{array}{cccc}
P_{11}^{d} & 0 & 0 & 0 \\
0 & P_{22}^{d} & 0 & 0 \\
0 & 0 & P_{33}^{d} & 0 \\
P_{41}^{d} & P_{42}^{d} & P_{43}^{d} & P_{44}^{d}
\end{array}\right)
$$

with $P_{11}^{d}=\left(P_{11}^{d}\right)^{T}>0, P_{22}^{d}=\left(P_{22}^{d}\right)^{T}>0$ and $P_{33}>0$. One can note that from (60) and (66), the Lyapunov function is quadratic in the system and controller states because $V\left(x_{a}(t)\right)=$ $x^{T}(t) P_{11}^{d} x(t)+x_{c}^{T}(t) P_{22}^{d} x_{c}(t)$.

Applying the same developments as for the static controller with the variable changes

$$
\left\{\begin{array}{l}
\bar{A}_{j}^{c}=P_{22}^{d} A_{j}^{c} \quad \bar{B}_{j}^{c}=P_{22}^{d} B_{j}^{c} \\
\bar{C}_{j}^{c}=\left(P_{33}^{d}\right)^{T} C_{j}^{c} \quad \bar{D}_{j}^{c}=\left(P_{33}^{d}\right)^{T} D_{j}^{c}
\end{array}\right.
$$

and defining $\varepsilon^{d}$ and $\delta^{d}$ by

$$
\begin{aligned}
\varepsilon^{d} & =\min _{i=1: 3^{n_{u}}, j=1: n} \lambda_{\min }\left(-\mathscr{Q}_{i j}^{d}\right) \\
\delta^{d} & =\max _{i=1: 3^{n u}, j=1: n} \Gamma_{i}^{T}\left(B_{j}^{T} \Sigma_{i j}^{1 d} B_{j}+D_{i}^{T} \Sigma_{i j}^{2 d} D_{i}\right) \Gamma_{i}
\end{aligned}
$$

with $\mathscr{Q}_{i j}^{d}$ defined in the same way as $\mathscr{2}_{i j}^{s}$ was, that is,

$$
\begin{aligned}
\mathscr{Q}_{i j}^{d}= & \mathbb{S}\left(\left(\mathscr{A}_{i j}^{d}\right)^{T} P^{d}\right)+\operatorname{diag}\left(P_{1}^{d}\left(\Sigma_{i j}^{1 d}\right)^{-1} P_{1}^{d}+\left(P_{41}^{d}\right)^{T}\left(\Sigma_{i j}^{2 d}\right)^{-1} P_{41}^{d},\right. \\
& \left.\left(P_{42}^{d}\right)^{T}\left(\Sigma_{i j}^{2 d}\right)^{-1} P_{42}^{d},\left(P_{43}^{d}\right)^{T}\left(\Sigma_{i j}^{2 d}\right)^{-1} P_{43}^{d},\left(P_{44}^{d}\right)^{T}\left(\Sigma_{i j}^{2 d}\right)^{-1} P_{44}^{d}\right)
\end{aligned}
$$

the stabilizing conditions are linearized and given by (62). As the weighting functions satisfy (2) and $\Sigma_{i j}^{1 d}, \Sigma_{i j}^{2 d}>0$, if (62) holds and $\left\|x_{a}\right\|_{2}^{2}>\frac{\delta^{d}}{\varepsilon^{d}}$, then $\dot{V}\left(x_{a}(t)\right)<0$, implying that $x_{a}(t)$ converges 
to an origin-centered ball of radius $\sqrt{\frac{\frac{8}{d}^{d}}{\varepsilon^{d}}}$. Similarly to the proof of Theorem 2, the radius of the ball is bounded by $\beta^{d}$ owing to (62) and is minimized in (61).

\section{Remark 2}

Compared with the state feedback control, the dynamic output feedback control introduces additional degrees of freedom in the controller design. Thus, the stability of the unsaturated open-loop subsystems (namely, the matrices $A_{i}$, for $i=1, \ldots, n$ ) is no longer required for the LMI constraints to be feasible.

\subsection{Numerical example}

Let us consider the same cart-pendulum system (40) in Section 4.3; both static and dynamic output feedback controllers are designed, and their performances are compared. The horizontal and angular velocity can be measured, and then the output matrices are defined by

$$
C=\left(\begin{array}{llll}
1 & 0 & 0 & 0 \\
0 & 0 & 0 & 1
\end{array}\right), D=\left(\begin{array}{l}
0 \\
0
\end{array}\right)
$$

From (70), the second measured output is $x_{4}(t)$, and then the premise variable $\xi(t)=x_{4}^{2}(t)$ is measurable, as assumed in the beginning of Section 2. Solving the optimization problem given in Theorem 2, the static controller gains are given by

$$
K_{1}^{s}=(-0.002-1.126), K_{2}^{s}=(-0.001-0.14)
$$

Applying Theorem 3 with $n_{c}=2$, the following dynamic controller gains are obtained:

$$
\begin{aligned}
& A_{1}^{c}=\left(\begin{array}{cc}
-0.324 & 0.083 \\
0.083 & -0.392
\end{array}\right), B_{1}^{c}=\left(\begin{array}{cc}
-0.003 & 0.002 \\
0.002 & -0.823
\end{array}\right) \\
& A_{2}^{c}=\left(\begin{array}{cc}
-0.332 & 0.783 \\
0.783 & -0.314
\end{array}\right), B_{2}^{c}=\left(\begin{array}{cc}
-0.003 & 0.002 \\
0.002 & -0.749
\end{array}\right) \\
& C_{1}^{c}=\left(\begin{array}{ll}
0.019 & -0.026
\end{array}\right), D_{1}^{c}=\left(\begin{array}{ll}
-0.002 & -0.0021
\end{array}\right) \\
& C_{2}^{c}=\left(\begin{array}{ll}
0.097 & 0.0127
\end{array}\right), D_{2}^{c}=\left(\begin{array}{ll}
-0.002 & -0.005
\end{array}\right)
\end{aligned}
$$
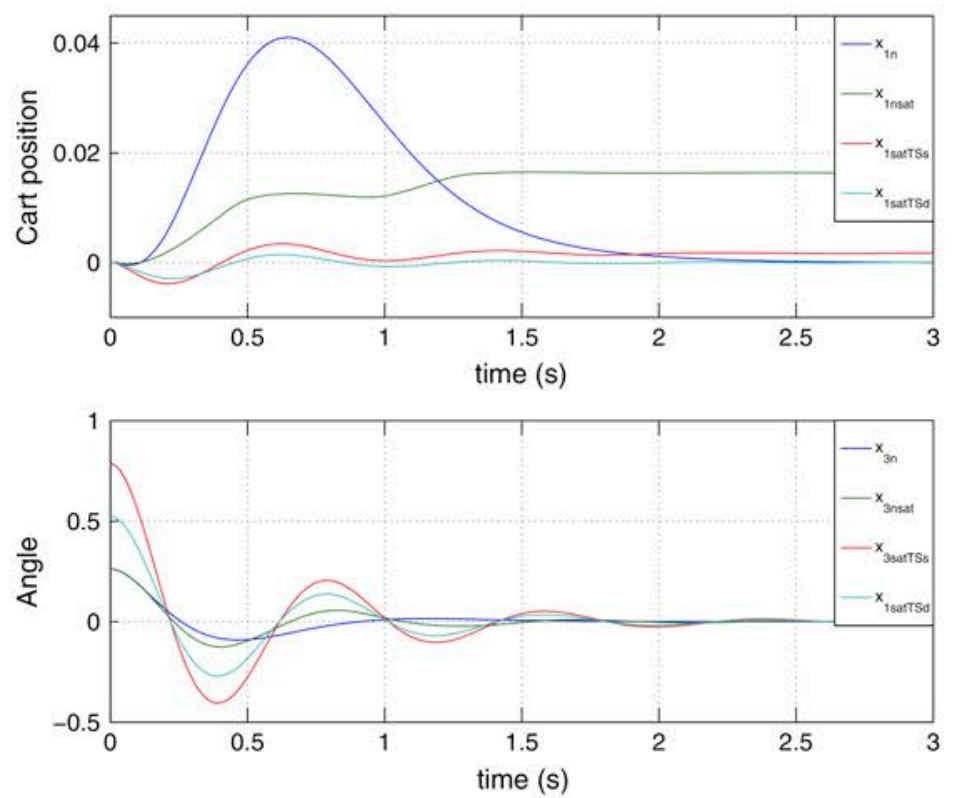

Figure 4. System states with output feedback control. 


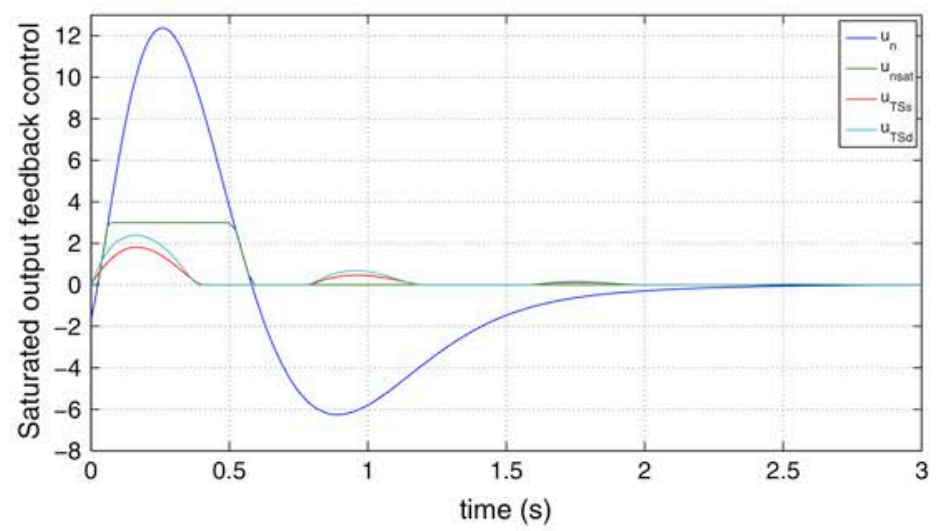

Figure 5. Saturated output feedback control.

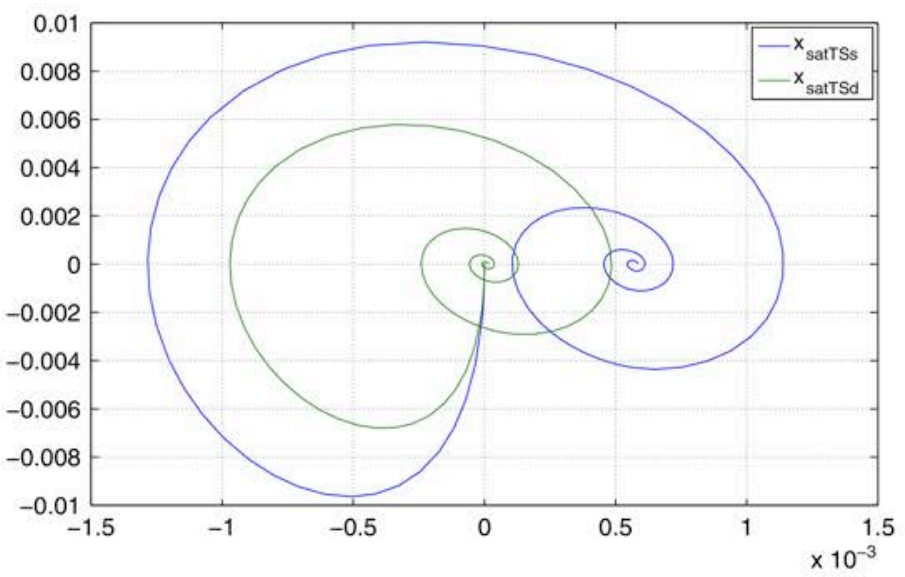

Figure 6. Phase diagram.

For the initial condition $x_{0}=\left(\begin{array}{ll}0 & 0 \pi / 120\end{array}\right)^{T}$, Figure 4 shows the system states in the following four cases. The system states $x_{N}$ and $x_{N, \text { sat }}$ are obtained from the nominal and saturated nominal controls presented in the previous example. The improvement from the proposed approach is also clear, because for the first state, the oscillation amplitudes are much smaller than the previous especially on the first state component. $x_{s a t, T S s}$ denotes the state trajectory for the proposed TS approach with static controller, and finally, $x_{s a t, T S d}$ stands for the proposed TS approach with dynamic controller.

From the depicted figures, it is clear that with the proposed TS approach, the convergence to an origin-centered ball is ensured. It is also clear that the obtained results with the dynamic controller are slightly better than the ones obtained with the static one, because the first state variable converges closer to the origin, and the oscillation damping of the second state variable is better.

The saturated control signals for each case are represented in Figure 5.

To compare the two controllers, in Figure6 are depicted the phase diagrams of the states $x_{2}$ and $x_{1}$ for both static and dynamic output controllers (the initial value of $\left(x_{1}, x_{2}\right)$ is $\left.(0,0)\right)$. One can see that applying the dynamic controller, the state converges to an origin-centered ball of radius $\beta^{d}=0.6 \times 10^{-5}$, which is smaller than $\beta^{s}=4.5 \times 10^{-3}$ obtained with a static controller.

\section{CONCLUSION AND FUTURE WORKS}

Thanks to a polytopic representation of the control input saturation, a nonlinear system with bounded inputs can be modeled as a TS system. It is important to note that using the proposed representation, 
the actuator model is expressed in terms of the control variables and of the saturation limits. As a consequence, these limits are taken into account when computing the controller gains. Moreover, the proposed TS approach allows to extend the use of linear tools, namely, the LMI formalism, to a nonlinear control problem.

A state feedback controller and both static and dynamic output feedback controllers were developed. The dynamic controller order is a degree of freedom fixed by the user. Relaxed LMI constraints are obtained with the use of the descriptor approach. The potentially destabilizing effect of the control saturation is compensated by the proposed controller ensuring that the closed-loop system state converges to an origin-centered ball.

In order to show the effectiveness of the proposed approach, all the results have been applied to the nonlinear model of a pendulum-cart with saturated actuator.

Future works may concern the extension of the present study to the TS models with unmeasurable premise variables (e.g., activating functions depending on the unmeasured state variables) by the mean of state observer-based controller. Another possible extension would be the use of more sophisticated Lyapunov functions (e.g., non-quadratic candidate functions) in order to obtain relaxed LMI constraints in the controller design procedure.

\section{REFERENCES}

1. Leonessa A, Haddad WM, Hayakawa T, Morel Y. Adaptive control for nonlinear uncertain systems with actuators amplitude and rate saturation constraints. International Journal of Adaptive Control and Signal Processing 2009; 23(1):73-96.

2. Fisher N, Kan Z, Kamalapurkar R, Dixon WE. Saturated RISE feedback control for a class of second-order nonlinear systems. IEEE Transactions on Automatic Control 2014; 59(4):1094-1099.

3. Zavala-Rio A, Santibaner V. A natural saturating extension of the PD with desired gravity compensation control law for robots manipulators with bounded inputs. IEEE Transactions on Robotics 2007; 23(2):386-391.

4. Kapoor N, Teel AR, Daoutidis P. An anti-windup design for linear systems with input saturation. Automatica 1999; 34(5):559-574.

5. Herrmann G, Menon PP, Turner MC, Bates DG, Postlethwaite I. Anti-windup synthesis for nonlinear dynamic inversion control schemes. International Journal of Robust and Nonlinear Control 2010; 20(13):1465-1482.

6. Biannic JM, Burlion L, Tarbouriech S, Garcia G. On dynamic inversion with rate limitations. American control conference, Montréal, Canada, 2012.

7. Zaccarian L, Teel AR. A common framework for anti-windup, bumpless transfer and reliable design. Automatica 2002; 38(10):1735-1744.

8. Mulder EF, Kothare MV, Morari M. Multivariable antiwindup controller synthesis using linear matrix inequalities. Automatica $2001 ; 37(9): 1407-1416$.

9. Grimm G, Hatfield J, Postlethwaite I, Teel AR, Turner MC, Zaccarian L. Antiwindup for stable linear systems with input saturation: an LMI-based synthesis. IEEE Transactions on Automatic Control 2003; 48(9):1509-1525.

10. Syaichu-Rohman A. Optimisation based feedback control of input constrained linear systems. Ph.D. Thesis, 2005.

11. Gomes da Silva Jr JM, Tarbouriech S. Antiwindup design with guaranteed regions of stability: an LMI-based approach. IEEE Transactions on Automatic Control 2005; 50(1):106-111.

12. Benzaouia A, Ouladsine M, Ananou B. Fault-tolerant saturated control for switching discrete-time systems with delays. International Journal of Adaptive Control and Signal Processing 2014; 29(10):1259-1273.

13. Scorletti G, El Ghaoui L. Improved LMI conditions for gain scheduling and related control problems. International Journal of Robust and Nonlinear Control 1998; 8(10):845-877.

14. Wu F, Grigoriadis KM, Packard A. Anti-windup controller design using linear parameter-varying control methods. International Journal of Control 2000; 73(12):1104-1114.

15. Zheng Q, Wu F. Output feedback control of saturated discrete-time linear systems using parameters dependent Lyapunov functions. System and Control letters 2008; 57:896-903.

16. Do AL, Gomes da Silva Jr. JM, Sename O, Dugard L. Control design for LPV systems with input saturation and state constraints: an application to a semi-active suspension. $50^{\text {th }}$ IEEEE Conference on Decision and Control, Orlando, Florida, USA, 2011; 3416-3421.

17. Alessandri A, Baglietto M, Battistelli G. On estimation error bounds for receding-horizon filters using quadratic boundedness. IEEE Transactions on Automatic Control 2004; 49(8):1350-1355.

18. Blanchini F, Miani S. Set-Theoretic Methods in Control. Birkhauser Boston, 2008.

19. Cao YY, Lin Z. Robust stability analysis and fuzzy-scheduling control for nonlinear systems subject to actuator saturation. IEEE Transactions on Fuzzy Systems 2003; 11(1):57-67.

20. Tarbouriech S, Garcia G, Gomes da Silva Jr. JM, Queinnec I. Stability and Stabilization of Linear Systems with Saturating Actuators London (ed.). Springer-Verlag: London, 2011.

21. Hu T, Lin Z. Composite quadratic Lyapunov functions for constrained control systems. IEEE Transactions on Automatic Control 2003; 48(3):440-450. 
22. De Dona JA, Moheimani SOR, Goodwin GC, Feuer A. Robust hybrid control incorporating over-saturation. System and Control letters 1999; 38:179-185.

23. Klug M, Castelan EB, Leite VJS, Silva LFP. Fuzzy dynamic output feedback control through nonlinear TakagiSugeno models. Fuzzy Sets and Systems 2015; 263:92-111.

24. Guerra TM, Kruszewski A, Vermeiren L, Tirmant H. Conditions of output stabilization for nonlinear models in the Takagi-Sugeno's form. Fuzzy Sets and Systems 2006; 157(9):1248 -1259.

25. Xie W. Improved $\mathcal{L} 2$ gain performance controller synthesis for Takagi-Sugeno fuzzy system. IEEE Transactions on Fuzzy Systems 2008; 16(5):1142-1150.

26. Tanaka K, Ohtake H, Wang HO. A descriptor system approach to fuzzy control system design via fuzzy Lyapunov functions. IEEE Transactions on Fuzzy Systems 2007; 15(3):333-341.

27. Guelton K, Bouarar T, Manamanni N. Robust dynamic output feedback fuzzy Lyapunov stabilization of TakagiSugeno systems - a descriptor redundancy approach. Fuzzy Sets and Systems 2009; 160(19):2796-2811.

28. Astorga-Zaragoza CM, Theilliol D, Ponsart JC, Rodrigues M. Fault diagnosis for a class of descriptor linear parameter-varying systems. International Journal of Adaptive Control and Signal Processing 2012; 26(3):208-223.

29. Tanaka K, Wang HO. Fuzzy Control Systems Design and Analysis: A Linear Matrix Inequality Approach. John Wiley \& Sons, Inc.: New York, 2001.

30. Boyd S, El Ghaoui L, Feron E, Balakrishnan V. Linear Matrix Inequalities in System and Control Theory, Studies in Applied Mathematics, vol. 15. Society for Industrial and Applied Mathematics (SIAM): Philadelphia, PA, 1994.

31. Bezzaoucha S, Marx B, Maquin D, Ragot J. Linear feedback control input under actuator saturation: a Takagi-Sugeno approach. 2nd International Conference on Systems and Control, ICSC'12, Marrakech, Morocco, 2012.

32. Zhang K, Jiang B, Shi P. A new approach to observer-based fault-tolerant controller design for Takagi-Sugeno fuzzy systems with state delay. Circuits, Systems, and Signal Processing 2009; 28(5):679-697.

33. Bezzaoucha S, Marx B, Maquin D, Ragot J. Contribution to the constrained output feedback control. American Control Conference, ACC, Washington, DC, USA, 2013; 235-240. 